\title{
EXTRACCIÓN Y BENEFICIO DE SEMILLA DE TOMATE DE CÁSCARA (Physalis ixocarpa Brot. ex Horm.)
}

\section{EXTRACTION AND PROCESSING OF HUSK TOMATO SEED (Physalis ixocarpa Brot. ex Horm.)}

\author{
Jorge Coronado-González', Aureliano Peña-Lomelí *, Natanael Magaña-Lira ${ }^{1}$, \\ Jaime Sahagún-Castellanos ${ }^{2}$ y Carmen Ybarra-Moncada ${ }^{3}$
}

\author{
'Universidad Autónoma Chapingo (UACh), Instituto de Horticultura. ${ }^{2}$ UACh, Departamento de Fitotecnia. ${ }^{3}$ UACh, Departamento de Ingeniería Agroin- \\ dustrial, Chapingo, Texcoco, Estado de México, México. \\ *Autor para correspondencia (aplomeli@correo.chapingo.mx)
}

\section{RESUMEN}

\begin{abstract}
La semilla de tomate de cáscara (Physalis ixocarpa Brot. ex Horm.) que se usa en México en general es de baja calidad física, fisiológica y genética. Para producir semilla de alta calidad es necesario contar con variedades mejoradas y tecnología adecuada para su producción y beneficio. Se compararon dos máquinas para la extracción de semilla (molino eléctrico y despulpadora) con el objetivo de estudiar su eficiencia y efecto sobre el rendimiento y calidad de semilla de cuatro variedades mejoradas de tomate de cáscara (Diamante, Tecozautla 04, Gema y Manzano Tepetlixpa). La investigación se llevó a cabo en Chapingo, Estado de México, de julio a octubre de 2013, con un arreglo de tratamientos factorial $2 \times 4$, bajo un diseño experimental completamente al azar con cuatro repeticiones. Se extrajo y benefició la semilla de $1000 \mathrm{~kg}$ de fruto por unidad experimental. Se evaluó el tiempo total del proceso de extracción, rendimiento de materia prima (semilla sin beneficio), peso de semilla seca clasificada por tamaño (chica, estándar y grande), contenido de humedad de la materia prima en tres etapas del proceso de secado y germinación de semilla en cuatro etapas del proceso de beneficio. Se obtuvo el menor tiempo total de proceso y el mayor rendimiento de semilla con la máquina despulpadora. Con ambas máquinas se obtuvo la misma calidad fisiológica de la semilla. Las variedades evaluadas produjeron semilla de calidad fisiológica similar, aunque con diferente rendimiento de semilla. Existe interacción significativa entre máquinas y variedades para rendimiento de materia prima y de semilla beneficiada, al igual que para germinación.
\end{abstract}

Palabras clave: Physalis ixocarpa, acondicionamiento de semilla, germinación, tamaño de semilla.

\section{SUMMARY}

The husk tomato (Physalis ixocarpa Brot. ex Horm.) seed used in Mexico is generally of low physical, physiological and genetic quality. In order to produce seeds of high quality, it is necessary to have improved varieties and the appropriate technology for its production and processing. Two machines for seed extraction (electric mill and pulper) were compared in order to study their efficiency and effect on seed yield and quality of four improved husk tomato varieties (Diamante, Tecozautla 04, Gema and Manzano Tepetlixpa). The research was carried out at Chapingo, State of Mexico, from July to October 2013, with a $2 \times 4$ factorial arrangement, under a completely randomized experimental design with four replications. The seed of $1000 \mathrm{~kg}$ of fruit per experimental unit was extracted and processed. The total time of the extraction process, raw material (unprocessed seed) yield, dry seed weight classified by size (small, standard and large), moisture content of the raw material in three stages of the drying process and seed germination in four stages of the processing were evaluated. The lowest total processing time and highest seed yield were obtained with the pulper machine. With both machines the same physiological seed quality was obtained. The evaluated varieties produced seeds of similar physiological quality, but different seed yield. There was significant interaction between machines and varieties for yield of raw material, yield of processed seed as well as for germination.

Index words: Physalis ixocarpa, seed processing, germination, seed size.

\section{INTRODUCCIÓN}

El tomate de cáscara (Physalis ixocarpa Brot. ex Horm.) ha adquirido gran relevancia en México en los últimos años. En la actualidad es el séptimo cultivo hortícola después del tomate (Solanum lycopersicum), chile (Capsicum annuum), papa (Solanum tuberosum), cebolla (Allium cepa), sandía (Citrullus lanatus) y pepino (Cucumis sativus). En 2017 se sembró en 26 estados de la República Mexicana en una superficie total de $43,172.56$ ha, con un rendimiento medio de $18.08 \mathrm{t} \mathrm{ha}^{-1}$. Destacan por su producción los estados de Sinaloa, Puebla, Jalisco, Zacatecas, Nayarit, Michoacán y Estado de México (SIAP, 2018).

El establecimiento del cultivo de tomate de cáscara puede ser por trasplante o siembra directa, para lo cual se requiere de 0.2 a $1.5 \mathrm{~kg}$ de semilla por hectárea, respectivamente. Debido a la magnitud de la superficie sembrada, la semilla tiene gran demanda; sin embargo, mucha de la ofrecida en el mercado es de baja calidad física, fisiológica y genética. Por lo anterior, es importante generar información y tecnologías para la producción y beneficio de la semilla, ya que la investigación al respecto en tomate de cáscara es escasa (Marín et al., 2007); además, es importante producir semilla de variedades registradas, para ofrecerlas con la calidad y en los volúmenes adecuados en las regiones agrícolas de producción, para contribuir a que los productores incrementen el rendimiento de fruto y se acerquen al potencial de $40 \mathrm{t} \mathrm{ha}^{-1}$ obtenido con variedades 
mejoradas (Peña-Lomelí et al., 2014).

En México la mayoría de las semillas de especies hortícolas es importada, por lo que es importante desarrollar la tecnología necesaria para producir semillas de hortalizas de alta calidad (Robledo et al., 2010). La calidad en las semillas se mide por cuatro parámetros principales: a) calidad física, que se encuentre libre de impurezas, material inerte y semillas dañadas; b) calidad fisiológica, dada por su viabilidad, capacidad germinativa y vigor; c) calidad genética, asociada con su potencial genético superior y su pureza varietal; y d) calidad sanitaria, que no posea ni sea vector de enfermedades o plagas. La calidad está en relación directa con el uso de la tecnología de semillas como herramienta fundamental para obtener dicha calidad, misma que se ve afectada por las condiciones en las cuales se produjo, así como por el proceso de beneficio (Ayala-Villegas et al., 2014; Doria, 2010).

Con el beneficio se busca obtener la máxima cantidad de semilla pura con el más alto grado de uniformidad, vigor y germinación, a un costo razonable; para lograrlo, la semilla se debe extraer adecuadamente, bajar el contenido de humedad, eliminar impurezas, clasificarla y protegerla contra plagas y enfermedades (Doria, 2010).

Para la extracción de semilla de frutos carnosos, como los del tomate de cáscara, la molienda debe ser realizada por una máquina despulpadora, donde un tornillo sin fin o una estructura de aspas permiten el rompimiento de los frutos, lo que genera una mezcla que se envía a tinas con agua para decantar impurezas y seleccionar la semilla más pesada (Martínez et al., 2004). La FAO (2011) menciona que el contenido de humedad de la semilla para su almacenamiento y venta es el factor más crítico que afecta la tasa de deterioro. Baudet et al. (1999) señalan que el secado es fundamental en la producción de semillas, ya que permite conservar la calidad inicial durante el almacenamiento.

La limpieza y clasificación constituyen etapas muy importantes en el beneficio de semillas y tienen por finalidad eliminar las impurezas, clasificar las semillas por tamaños para la siembra y por medio de la separación de las semillas dañadas o deterioradas elevar la calidad, independientemente de sus características genéticas (Doria, 2010).

La producción de fruto de tomate de cáscara se realiza en riego o temporal. Su establecimiento en campo es por siembra directa o transplante. En siembra directa, sobre todo en condiciones de temporal, se emplean de 1 a $2 \mathrm{~kg}$ de semilla por hectárea y ésta es producida por los propios agricultores. El proceso consiste en macerar o moler frutos maduros cosechados en la propia parcela, separar la pulpa gruesa y la cáscara del fruto de la semilla y la pulpa fina, depositar la mezcla de semilla y pulpa fina en un recipiente con agua, eliminar por decantación la semilla vana y la pulpa fina, colectar la semilla asentada en el fondo del recipiente y ponerla a secar al sol. Esta semilla, que generalmente tiene bajo porcentaje de germinación, es la que el productor siembra (Magaña et al., 2011).

En la Universidad Autónoma Chapingo (UACh), desde 1985 se desarrolla un programa de mejoramiento genético de tomate de cáscara. Como resultado del mismo, a la fecha se han registrado 11 variedades mejoradas y se cuenta con dos máquinas para la extracción de semilla, una diseñada por investigadores de la propia universidad (molino eléctrico) y una despulpadora adaptada para extraer semilla de la especie, que utiliza la toma de fuerza del tractor (Peña-Lomelí et al., 2014; SNICS, 2018). A la fecha no se ha evaluado la eficiencia de las máquinas en la extracción de semilla y se desconoce el rendimiento de semilla de las variedades.

En el presente estudio se evaluaron dos máquinas extractoras de semilla en cuatro variedades de tomate de cáscara, con el objetivo de determinar los tiempos del proceso de extracción de semilla en cada máquina y evaluar la calidad fisiológica y rendimiento de semilla antes y después de su beneficio y clasificación por tamaño.

\section{MATERIALES Y MÉTODOS}

\section{Material genético y sitio experimental}

Se cosechó fruto maduro. Las variedades Diamante, Tecozautla 04 y Gema fueron producidas en el ciclo primavera-verano 2013 en el Campo Agrícola Experimental de la Universidad Autónoma Chapingo (UACh), en Chapingo, Texcoco, Estado de México. La variedad Manzano Tepetlixpa se cultivó en el Campo Experimental del Colegio de Postgraduados, en Montecillo, Texcoco, Estado de México en el mismo ciclo agrícola. La fase de extracción y beneficio de los lotes de semilla se realizó durante el mes de julio del mismo año en el área de Semillas del Campo Experimental de la UACh, mientras que en septiembre y octubre se realizaron las pruebas de calidad de semilla en los Laboratorios de Ecología y de Análisis de Semillas del Departamento de Fitotecnia de la UACh.

\section{Estructura del experimento}

Se incluyeron dos factores de estudio, el primero de ellos fue el tipo de máquinas extractoras de semilla con dos niveles: una despulpadora de molino fija, accionada 
por un motor eléctrico (M1: Eléctrica) y una despulpadora móvil de molino y tambor accionada con la toma de fuerza de un tractor (M2: Despulpadora). El segundo factor fue variedades, con cuatro poblaciones de tomate de cáscara (Diamante, Tecozautla 04, Gema y Manzano Tepetlixpa). Se usó un arreglo de tratamientos factorial completo $2 \times 4$, producto de la combinación de las dos máquinas extractoras y las cuatro variedades de tomate de cáscara, lo cual generó ocho tratamientos. El diseño experimental fue completamente al azar con cuatro repeticiones. Cada unidad experimental fue de $1000 \mathrm{~kg}$ de fruto procesado. La evaluación de los tratamientos se realizó en tres etapas, la primera durante el proceso de extracción, la segunda durante el secado y la tercera después de la limpieza y clasificación de la semilla.

\section{Variables evaluadas}

Para la primera etapa se cosecharon frutos de las cuatro variedades en madurez fisiológica, 90 días después del trasplante y 60 días después de la polinización (Pérez et al., 2008). Se pesaron lotes de $1000 \mathrm{~kg}$ con una báscula Torrey ${ }^{\circledR}$ (México) con capacidad para 500 kg. Para no contaminar los lotes de cada variedad, se procesó una variedad cada día y las máquinas se limpiaron con agua a presión después de terminado el proceso de cada variedad. Para cada unidad experimental (UE) se determinó el tiempo total de proceso (TTP) de extracción en min, desde que se inició la molienda del fruto hasta que se obtuvo el total de semilla de la UE. EI TTP incluyó el tiempo de molienda, de cepillado, de decantado y de cribado en el tren de lavado (Martínez et al., 2004). La semilla extraída de cada UE fue colectada en cajas de plástico con capacidad de $25 \mathrm{~kg}$. El peso de semilla húmeda (PSH) en kg se determinó en una báscula digital marca Torrey®, con precisión de $5 \mathrm{~g}$.

La segunda etapa abarcó desde que terminó la extracción hasta que culminó el secado de la semilla. Después de la extracción, la semilla pasó a un proceso de centrifugado en una máquina Blue Point ${ }^{\circledR}$ (Kenosha, Wisconsin, EUA) a 3200 rpm para eliminar exceso de agua. La semilla centrifugada fue llevada al área de secado al ambiente, donde se colocó en bastidores metálicos de $1.9 \mathrm{~m}$ de largo $\times 1.3 \mathrm{~m}$ de ancho, con capacidad para $10 \mathrm{~kg}$ de semilla, distribuida uniformemente en una lámina de $1 \mathrm{~cm}$ de espesor, colocados a $50 \mathrm{~cm}$ sobre el piso para garantizar la circulación de aire. El secado tardó dos d. Cada lote de semillas por unidad experimental se colocó en cubetas de plástico de $19 \mathrm{~L}$ y se registró el peso $(\mathrm{kg})$ de semilla seca sin beneficiar (PSSSB) con una báscula marca Torrey ${ }^{\circledR}$ con precisión de $1 \mathrm{~g}$.

De la semilla recién extraída, centrifugada y seca se tomaron muestras de $15 \mathrm{~g}$ por UE, que se resguardaron en cajas de Petri de plástico y se llevaron al laboratorio, donde para cada UE se pesó una sub-muestra de $5 \mathrm{~g}$ en una balanza analítica Oahus Pioneer ${ }^{\circledR}$ (Oahus Corp, Parsippany, New Jersey, EUA) y una sub sub-muestra de 100 semillas. Las sub-muestras de $5 \mathrm{~g}$ fueron colocadas en cajas de Petri de vidrio e introducidas en una estufa de secado StableTemp 52120-02 (Cole Parmer ${ }^{\circledR}$ Instrument Company, Vernon Hills, Illinois, EUA) a $72{ }^{\circ} \mathrm{C}$ durante 72 h (Pérez et al., 2012). Posteriormente, se les cuantificó la masa final y se calculó porcentaje de humedad después del despulpado (HDD), después del centrifugado (HDC) y después del secado (HDS), mediante la fórmula de Bewley y Black (1994)

Cada sub sub-muestra de 100 semillas se usó para realizar pruebas de germinación. Se usaron cajas de Petri de plástico transparente de $95 \mathrm{~mm}$ de diámetro por $10 \mathrm{~mm}$ de profundidad. La semilla se colocó distribuida sobre papel filtro de poro mediano humedecido con 5 $\mathrm{mL}$ de agua destilada. Se usó una cámara germinadora Seedburo® D-7140 (Seedburo Equipment Company, Des Plaines, llinois, EUA.) a $30 \pm 1{ }^{\circ} \mathrm{C}$ y $90 \%$ de humedad relativa. La prueba se realizó en oscuridad durante $21 \mathrm{~d}$, con cuatro repeticiones de 100 semillas, de acuerdo con las normas de la ISTA (2004), y mediante la misma se evaluó la germinación (\%) después del despulpado (GDD), después del centrifugado (GDC) y después del secado sin clasificar (GDS).

Para la tercera etapa se eliminaron manualmente los grumos de semilla seca y con un harnero de malla de 60 $\times 45 \mathrm{~cm}$, con perforaciones de $4 \mathrm{~mm}$, se eliminaron las impurezas mayores (restos de tallos, hojas y cáscaras del fruto). Posteriormente, la semilla se sometió a un proceso de clasificado por tamaño con una máquina de aire y zarandas tipo Seedburo ${ }^{\circledR}$ modelo LA-LS/B. Para obtener el tamaño chico (C1), estándar (C2) y grande (C3) se usaron cribas de 1.5, 2.0 y 3.0 mm de diámetro, respectivamente. En esta operación fueron separadas las impurezas menores como tierra, y mayores como basura, además de que se eliminó la semilla vana. Se realizó un segundo paso de proceso de clasificación de la semilla de tamaño estándar.

Con una báscula digital marca Torrey ${ }^{\circledR}$ con capacidad de $15 \mathrm{~kg}$ y precisión de $1 \mathrm{~g}$, se obtuvieron los pesos de semilla seca beneficiada y clasificada por tamaños chico (PSSBC 1), estándar (PSSBC2) y grande (PSSBC3). De cada tamaño de semilla por UE se tomaron muestras de 100 semillas, que fueron llevadas al laboratorio para realizar las pruebas de germinación, siguiendo el procedimiento descrito en la etapa anterior, con el fin de cuantificar la germinación después del secado, beneficiado y clasificado en tamaños chico (GDSBC1), estándar (GDSBC2) y grande (GDSBC3). 


\section{Análisis estadístico}

Se realizaron análisis de varianza y pruebas de comparación de medias de Tukey $(P \leq 0.05)$ con el paquete estadístico SAS versión 9.1 (SAS Institute, 2002). Para las variables donde la interacción Máquinas $\times$ Variedades fue significativa, se realizaron comparaciones de medias de Tukey para variedades en cada máquina por separado.

\section{RESULTADOS Y DISCUSIÓN}

\section{Factor de variación máquinas}

En el análisis de varianza se detectaron diferencias significativas $(P \leq 0.05)$ entre máquinas para las variables TTP, PSSBC2 y PSSBC3, donde la máquina despulpadora tuvo el mejor desempeño (Cuadro 1). El TTP de la despulpadora fue $40 \%$ menor con respecto al de máquina eléctrica, lo cual se debe a que la máquina despulpadora separa la cáscara y la pulpa gruesa de la semilla y la pulpa fina, mientras que en la máquina eléctrica se obtiene una mezcla de cáscara, pulpa gruesa, pulpa fina y semilla, que debe ser separada manualmente en el tren de lavado. También se obtuvo mayor cantidad de semilla de tamaño estándar y grande con la máquina despulpadora, lo que puede deberse a que para la máquina eléctrica una porción significativa de semilla mediana y grande se queda adherida a la pulpa gruesa y la cáscara durante la separación manual en el tren de lavado.

Martínez et al. (2004) señalaron que la clasificación de la semilla de tomate de cáscara incrementa su porcentaje de germinación; además, aseguran que el tamaño de semilla medio (estándar) tiene mejor calidad y se obtiene en mayor cantidad, lo cual coincide con lo encontrado en la presente investigación para la variable PSSBC2, que corresponde a la cantidad de semilla estándar; sin embargo, para el porcentaje de germinación no se encontraron diferencias entre las máquinas (Cuadro 1). Para el caso de ambas máquinas, la proporción de tamaños después de la clasificación fue la misma: 26 \% chica, 73 \% estándar y 1 $\%$ grande.

\section{Factor variedades}

Para el factor variedades, en el análisis de varianza se detectaron efectos significativos $(P \leq 0.05)$ sobre 12 de las 15 variables evaluadas. Sólo para GDC, GDSBC2 y GDSBC3 no se encontró significancia estadística.

En la prueba de comparación de medias entre variedades (Cuadro 2) se observa que Tecozautla 04 tuvo el menor TTP, sólo diferente $(P \leq 0.05)$ al de Gema, que fue el mayor, ésto debido a que la estandarización del proceso de extracción de la semilla se ha desarrollado principalmente en la variedad Tecozautla 04, que es de frutos firmes, en contraste con Gema, que es de frutos duros, no obstante que ambas son de fruto muy grande (Peña et al., 2011).

La variedad Diamante presentó mayor rendimiento de semilla extraída para las cinco variables de peso (PSH, PSSSB, PSSBC1, PSSBC2 y PSSBC3), aunque para PSSBC2 y PSSBC3 no fue diferente de Tecozautla 04 y Gema. En contraste, la variedad Manzano Tepetlixpa tuvo en las mismas cinco variables el menor rendimiento, excepto que en PSH no fue estadísticamente diferente a Tecozautla 04. Aunque estas dos variedades rindieron la misma cantidad de materia prima (PSH), Manzano Tepetlixpa presenta mayor humedad después del despulpado (HDD), por lo que al final presenta menor rendimiento de semilla seca beneficiada, mientras que en PSSBC3 no fue superada por Gema ni Tecozautla 04.

La variedad Manzano Tepetlixpa pertenece a la raza Manzano, en tanto que Tecozautla 04 y Gema pertenecen a la raza Puebla, y Diamante proviene de un híbrido intervarietal de las razas Rendidora y Puebla (Peña-Lomelí et al., 2014). Al respecto, Martínez et al. (2004) refieren que el rendimiento de semilla varía en función de la raza a la que pertenece la variedad, lo cual coincide con los resultados aquí obtenidos.

Para las variables de humedad de la semilla (Cuadro 2) se observa que Manzano Tepetlixpa presentó el mayor contenido de humedad de la semilla durante el proceso de extracción en los diferentes puntos de muestreo (HDD, HDC y HDS), lo cual puede deberse a su mayor tamaño de semilla (Peña etal., 2011). Para HDD, Diamante y Tecozautla 04 presentaron el menor contenido de humedad, así como la variedad Gema en HDS. De lo anterior se deduce que, al tener mayor contenido de humedad, Manzano Tepetlixpa requiere de mayor tiempo de secado con respecto al resto de las variedades, lo cual es relevante en el proceso de beneficio de semilla. Como era de esperarse, el centrifugado y el secado redujeron la humedad de la semilla, que después del despulpado era de 48 a $62 \%$ y después del secado se ubicó entre 5.1 y 7.8 \% en HDS, lo que mejora su calidad. Estos datos son relevantes porque en tomate de cáscara no existe información precisa con respecto a los contenidos de humedad en cada paso del proceso de extracción y secado de la semilla.

En relación con la germinación antes del beneficio, Diamante, Tecozautla 04 y Gema tuvieron el mayor valor (GDD), pero sólo ésta última superó $(P \leq 0.05)$ a Manzano Tepetlixpa (Cuadro 2). La variedad Diamante presentó la mayor GDS, aunque sólo superó estadísticamente a Manzano Tepetlixpa. Estos datos son relevantes en la 
Cuadro 1. Comparación de medias de 15 variables de calidad física y fisiológica utilizando dos máquinas de extracción de semilla de tomate de cáscara (Physalis ixocarpa Brot. ex Horm.)

\begin{tabular}{|c|c|c|c|c|c|}
\hline Máquina & TTP (min) & PSH (kg) & PSSSB (kg) & HDD (\%) & HDC (\%) \\
\hline Eléctrica & $105.53 \mathrm{a}^{+}$ & $23.54 a$ & 9.36 a & $52.73 \quad a$ & 40.99 a \\
\hline Despulpadora & 61.78 b & $23.63 \mathrm{a}$ & 9.64 a & $53.46 \quad a$ & $41.35 \mathrm{a}$ \\
\hline \multirow[t]{2}{*}{ DSH 0.05} & 6.21 & 0.94 & 0.48 & 1.80 & 1.13 \\
\hline & HDS (\%) & GDD (\%) & GDC (\%) & GDS (\%) & PSSBC1 (g) \\
\hline Eléctrica & $6.21 \mathrm{a}$ & 35.19 a & $31.00 \mathrm{a}$ & $36.13 \mathrm{a}$ & 1915.1 a \\
\hline Despulpadora & 6.43 a & 36.06 a & $33.17 \mathrm{a}$ & 38.63 a & 2044.2 a \\
\hline \multirow[t]{2}{*}{ DSH 0.05} & 0.24 & 8.12 & 8.41 & 5.22 & 203.5 \\
\hline & PSSBC2 (g) & PSSBC3 (g) & GDSBC1 (\%) & GDSBC2 (\%) & GDSBC3 (\%) \\
\hline Eléctrica & $5380.1 \quad b$ & 78.6 b & 88.13 a & 93.44 a & 89.19 a \\
\hline Despulpadora & 5854.8 a & 96.3 a & $93.31 \quad a$ & 95.63 a & 88.00 \\
\hline DSH 0.05 & 417.7 & 15.5 & 6.11 & 3.07 & 6.37 \\
\hline
\end{tabular}

${ }^{\dagger}$ Medias con letras iguales en cada columna dentro de cada una de las variables no son estadísticamente diferentes (Tukey, 0.05), TTP: tiempo total de proceso, PSH: peso de semilla húmeda, PSSSB: peso de semilla seca sin beneficiar, HDD: humedad después del despulpado, HDC: humedad después del centrifugado, HDS: humedad después del secado, GDD: germinación después del despulpado, GDC: germinación después del centrifugado, GDS: germinación después del secado, PSSBC1: peso de semilla seca beneficiada tamaño chico, PSSBC2: peso de semilla seca beneficiada tamaño estándar, PSSBC3: peso de semilla seca beneficiada de tamaño grande, GDSBC1: germinación después de secado y beneficiado en tamaño chico, GDSBC2: germinación después de secado y beneficiado en tamaño estándar, GDSBC3: germinación después de secado y beneficiado en tamaño grande, DSH: diferencia significativa honesta.

planeación del proceso de producción de semilla, pues las variedades difieren en la calidad de su materia prima; además, es común que los agricultores extraigan su propia semilla y la siembren sin mediar beneficio alguno, llegando a sembrar hasta $2 \mathrm{~kg}$ de semilla por hectárea debido a la baja germinación (Magaña et al., 2011). En el caso particular de Manzano Tepetlixpa, su mayor tamaño de semilla, menor número de semillas por fruto y ciclo más largo (Peña et al., 2011) son características que influyen en la menor calidad de la materia prima.

Después del beneficio sólo hubo diferencias en calidad fisiológica para la variable GDSBC1, donde la variedad Tecozautla 04 no superó estadísticamente a Diamante ni a Gema, pero fue superior a Manzano Tepetlixpa. En las cuatro variedades la germinación fue mayor en la semilla de tamaño estándar (GDSBC2) con respecto a los otros dos tamaños, aspecto importante porque es en esta categría donde se obtiene el mayor rendimiento de semilla (PSSBC2).

Los valores encontrados en porcentaje de humedad de la semilla en las variedades Tecozautla 04 y Diamante, al momento de su extracción, se aproximan al valor que encontraron Pérez et al. (2008), quienes refieren que la semilla de tomate de cáscara a los 63 días después de floración alcanza un punto de equilibrio a $45 \%$ en su contenido de humedad. Los contenidos de humedad encontrados al finalizar el proceso de secado de la semilla (de 5.1 a $7.8 \%$ ) son inferiores al reportado por Martínez et al. (2004), quienes señalan que después de un proceso de secado a la sombra por 3 d, la semilla de tomate de cáscara alcanza 11 \% de humedad. La diferencia puede deberse a que en la presente investigación el secado se hizo al sol durante $2 \mathrm{~d}$, aunque no se registraron la temperatura ni la humedad relativa, aspectos que serían importantes de estudiar.

La variedad Manzano Tepetlixpa presentó los menores porcentajes de germinación para dos (C1 y C2) de los tres tamaños de semilla, pero sólo en $\mathrm{C} 1$ fue estadísticamente inferior a la variedad Tecozautla 04, lo que indica que las cuatro variedades tuvieron una calidad fisiológica similar, estimada por el porcentaje de germinación. Después del proceso de clasificado de la semilla en tamaños chico (C1), estándar (C2), y grande (C3), todas las variedades, excepto la semilla chica de la variedad Manzano Tepetlixpa, tuvieron una germinación superior a $85 \%$, que es el mínimo requerido en semillas para siembra en general (FAO, 2011); sin embargo, para el caso de tomate de cáscara en México se requiere al menos 90 \% de germinación (SNICS, 2014), por lo que sólo es posible comercializar la semilla estándar de las cuatro variedades, la semilla chica de Diamante, Tecozautla 04 y Gema, y la semilla grande de Diamante (Cuadro 2). 
Cuadro 2. Comparación de medias de 15 variables de calidad física y fisiológica de semilla en cuatro variedades de tomate de cáscara (Physalis ixocarpa Brot. ex Horm.).

\begin{tabular}{|c|c|c|c|c|c|}
\hline Variedad & TTP (min) & $\mathrm{PSH}(\mathrm{kg})$ & PSSSB (kg) & $\operatorname{HDD}(\%)$ & $\operatorname{HDC}(\%)$ \\
\hline Diamante & $80.52 a^{a+}$ & 26.01 a & $10.98 \quad$ a & $48.07 \mathrm{C}$ & - \\
\hline Tecozautla 04 & 78.48 b & $22.15 \mathrm{C}$ & $9.26 \mathrm{~b}$ & $49.02 \mathrm{c}$ & $37.67 \mathrm{~b}$ \\
\hline Gema & 90.80 a & $24.15 b$ & $9.84 \mathrm{~b}$ & $53.11 \mathrm{~b}$ & $38.76 \mathrm{~b}$ \\
\hline Manzano Tepetlixpa & $84.82 a b$ & $22.03 \mathrm{C}$ & $7.90 \mathrm{c}$ & $62.17 \quad a$ & $47.08 \mathrm{a}$ \\
\hline \multirow[t]{2}{*}{ DSH 0.05} & 11.89 & 1.77 & 0.91 & 3.40 & 1.69 \\
\hline & HDS (\%) & GDD (\%) & GDC (\%) & GDS (\%) & PSSBC1 (g) \\
\hline Diamante & $6.39 \mathrm{~b}$ & $40.50 \mathrm{ab}$ & - & 43.75 a & 3264.9 a \\
\hline Tecozautla 04 & $6.01 \mathrm{~b}$ & $33.38 a b$ & 29.38 a & $37.88 \mathrm{ab}$ & $1752.1 \mathrm{C}$ \\
\hline Gema & $5.09 \mathrm{c}$ & 42.38 a & $35.63 \mathrm{a}$ & $37.75 \mathrm{ab}$ & 2409.9 b \\
\hline Manzano Tepetlixpa & 7.78 a & $26.25 b$ & $31.25 \mathrm{a}$ & $30.13 \mathrm{~b}$ & $491.8 \mathrm{~d}$ \\
\hline \multirow[t]{2}{*}{ DSH 0.05} & 0.45 & 15.35 & 12.51 & 9.87 & 384.7 \\
\hline & PSSBC2 (g) & PSSBC3 (g) & GDSBC1 (\%) & GDSBC2 (\%) & GDSBC3 (\%) \\
\hline Diamante & 6303.0 a & 103.6 a & $93.25 \mathrm{ab}$ & 94.50 a & $92.50 \mathrm{a}$ \\
\hline Tecozautla 04 & 6767.5 a & $91.6 \mathrm{ab}$ & 96.38 a & 96.50 a & 88.00 a \\
\hline Gema & 6445.5 a & $86.3 \mathrm{ab}$ & $90.75 \mathrm{ab}$ & $96.13 \mathrm{a}$ & 86.38 a \\
\hline Manzano Tepetlixpa & $2553.8 b$ & 68.3 b & $82.5 \mathrm{~b}$ & $91.00 \quad$ a & $87.50 \mathrm{a}$ \\
\hline DSH 0.05 & 789.5 & 29.4 & 11.56 & 5.81 & 12.04 \\
\hline
\end{tabular}

${ }^{\dagger}$ Medias con letras iguales en cada columna no son estadísticamente diferentes (Tukey, 0.05), TTP : tiempo total de proceso, PSH: peso de semilla húmeda, PSSSB: peso de semilla seca sin beneficiar, HDD: humedad después del despulpado, HDC: humedad después del centrifugado, HDS: humedad después del secado, GDD: germinación después del despulpado, GDC: germinación después del centrifugado, GDS: germinación después del secado, PSSBC1: peso de semilla seca beneficiada tamaño chico, PSSBC2: peso de semilla seca beneficiada tamaño estándar, PSSBC3: peso de semilla seca beneficiada tamaño grande, GDSBC1: germinación después del secado y beneficiado en tamaño chico, GDSBC2: germinación después del secado y beneficiado en tamaño estándar, GDSBC3: germinación después del secado y beneficiado en tamaño grande, DSH: diferencia significativa honesta.

\section{Interacción máquinas $\times$ variedades}

La interacción máquinas $\times$ variedades mostró significancia estadística $(P \leq 0.05)$ para las variables TTP, PSH, PSSSB, PSSBC3, GDSBC2 y GDSBC3. En la Figura 1 se muestra gráficamente el comportamiento diferencial de las variedades en cada máquina de extracción para estas seis características.

En la Figura 1 A se observa que, para la máquina eléctrica, Tecozautla 04 presenta el menor TTP, estadísticamente igual a Diamante, pero diferente de Gema y Manzano Tepetlixpa, mientras que en la máquina despulpadora no se observaron diferencias entre variedades, lo cual indica que es eficiente independientemente de la variedad; en contraste, en la máquina eléctrica es necesario hacer los ajustes en la planeación de la extracción en función de la variedad.

Para PSH la variedad mayormente involucrada en la interacción es Gema (Figura 1B), ya que en la máquina eléctrica se obtiene poca semilla, mientras que en la máquina despulpadora iguala a Diamante con la mayor cantidad obtenida. Esto se debe posiblemente a que Gema es una variedad de frutos duros, por lo que la máquina eléctrica no los tritura adecuadamente durante el tiempo evaluado y la semilla se pierde inmersa en la pulpa gruesa durante el lavado.

Manzano Tepetlixpa presentó el menor valor de PSSSB en la máquina eléctrica (Figura 1C), sin ser diferente estadísticamente de Tecozautla 04, mientras que en la máquina despulpadora dicha variedad tuvo el menor valor. En la variable PSSBC3 se presentó un comportamiento similar, con una reducción significativa para Manzano Tepetlixpa en la máquina despulpadora (Figura 1D). Lo anterior posiblemente se debe a que esta variedad tiene menor cantidad de jugo y su semilla se pierde en la pulpa gruesa y la cáscara, que son desechadas por la máquina despulpadora, que funciona con el jugo de los frutos. Por lo tanto, para extraer semilla de la variedad Manzano Tepetlixpa es necesario agregar agua a la despulpadora, 

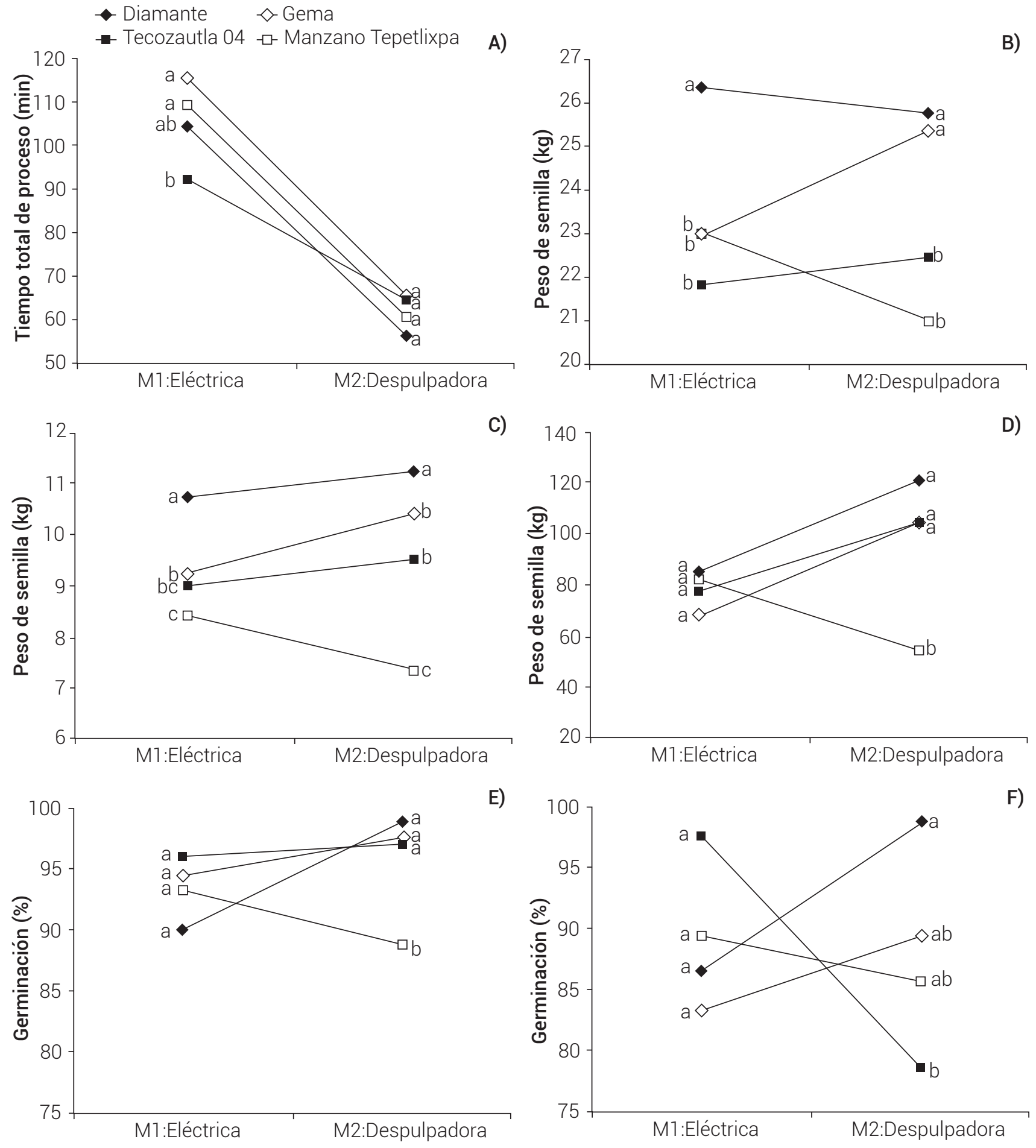

Figura 1. Comportamiento de cuatro variedades de tomate de cáscara con dos máquinas para extracción de semilla. A) tiempo total de proceso (TTP), B) peso de semilla húmeda (PSH), C) peso de semilla seca sin beneficiar (PSSSB), D) peso de semilla seca beneficiada tamaño grande (PSSBC3), E) germinación después del secado y beneficiado en tamaño estándar (GDSBC2), F) germinación después del secado y beneficiado en tamaño grande (GDSBC3). Medias con la misma letra para cada máquina no son estadísticamente diferentes (Tukey, 0.05). 
aunque se requieren estudios adiconales para confirmar lo anterior.

En las variables GDSBC2 y GDSBC3 no existieron diferencias significativas entre las variedades con el uso de la máquina eléctrica (Figuras 1 E y F). Para la máquina despulpadora la variedad Manzano Tepetlixpa fue estadísticamente inferior a las demás en GDSBC2. En la variable GDSBC3 la variedad Tecozautla 04 fue la de menor valor, pero sólo diferente de Diamante. Estos resultados indican que es necesario estudiar con mayor precisión las variedades señaladas cuando la extracción se realiza con la despulpadora, ya que las características varietales influyen significativamente en la calidad de la semilla (Martínez et al., 2004; Peña-Lomelí et al., 2014).

\section{CONCLUSIONES}

Se obtuvo mayor rendimiento de semilla beneficiada con la máquina despulpadora y el tiempo de extracción de ésta se redujo casi a la mitad con respecto al utilizado con la máquina eléctrica. Con ambas máquinas se obtiene la misma calidad fisiológica de semilla. Las variedades produjeron semilla de calidad fisiológica similar, aunque con diferente rendimiento. Existe interacción entre máquinas y variedades para tiempo total del proceso de extracción, rendimiento de materia prima, rendimiento de semilla beneficiada y germinación.

\section{BIBLIOGRAFÍA}

Ayala-Villegas M. J., O. J. Ayala-Garay, V. H. Aguilar-Rincón y T. Corona-Torres (2014) Evolución de la calidad de semilla de Capsicum annuum L. durante su desarrollo en el fruto. Revista Fitotecnia Mexicana 37:79-87.

Baudet L. M. L., F. A. Villela e C. Cavariani (1999) Princípios de secagem. Seed News 3:20-27.

Bewley J. D. and M. Black (1994) Seeds, Physiology of Development and Germination. Plenum Press. New York, USA. 445 p.

Doria J. (2010) Generalidades sobre las semillas: su producción, conservación y almacenamiento. Cultivos Tropicales 31:74-85.

FAO, Organización de las Naciones Unidas para la Alimentación y la Agricultura (2011) Semillas en Emergencias. Manual Técnico. Estudio FAO Producción y Protección Vegetal 202. Organización de las Naciones Unidas para la Alimentación y la Agricultura. Roma,
Italia. 83 p.

ISTA, International Seed Testing Association (2004) International Seed Testing Rules. International Seed Testing Association. Zurich Switzerland. $243 p$

Magaña L. N., J. F. Santiaguillo H. y 0. Grimaldo J. (2011) El mejoramiento participativo de tomate de cáscara como estrategia de conservación in situ. Folleto Técnico Número 48. Instituto Nacional de Investigaciones Forestales, Agrícolas y Pecuarias. Coatlinchán, Texcoco, Estado de México. 24 p.

Marín S. J., J. A. Mejía C., A. Hernández L., A. Peña L. y A. Carballo C. (2007) Acondicionamiento osmótico de semillas de tomate de cáscara. Agricultura Técnica en México 33:115-123.

Martínez S. J., A. Peña L. y D. Montalvo H. (2004) Producción y tecnología de semilla de tomate de cáscara. Boletín Técnico No. 4. Departamento de Fitotecnia, Universidad Autónoma Chapingo Chapingo, Estado de México. 35 p.

Peña L. A., N. Magaña L., S. Montes H., J. Sánchez M., J. F. Santiaguillo H., 0. Grimaldo J. y A. Contreras R. (2011) Manual Gráfico para la Descripción Varietal de Tomate de Cáscara (Physalis ixocarpa Brot. ex Horm.). Servicio Nacional de Inspección y Certificación de Semillas, Secretaria de Agricultura, Ganadería, Desarrollo Rural, Pesca y Alimentación-Universidad Autónoma Chapingo. Chapingo, Estado de México. 90 p.

Peña-Lomelí A., J. J. Ponce-Valerio, F. Sánchez-del-Castillo y N. MagañaLira (2014) Desempeño agronómico de variedades de tomate de cáscara en invernadero y campo abierto. Revista Fitotecnia Mexicana 37:381-391

Pérez C. I., V. A. González H., J. C. Molina M., 0. J. Ayala G. y A. Peña L. (2008) Efecto de desarrollo y secado de semillas de Physalis ixocarpa Brot. en germinación, vigor y contenido de azúcares. Interciencia 33:762-766

Pérez C. I., V. A. González H., 0. J. Ayala G., J. A. Carrillo S., G. García S., A. Peña L. y E. Cruz C. (2012) Calidad fisiológica de semillas de Physalis ixocarpa en función de madurez a cosecha y condiciones de almacenamiento. Revista Mexicana de Ciencias Agrícolas 3:67-78, https://doi.org/10.29312/remexca.v3i1.1481

Robledo T. V., M. M. Ramírez G., M. E. Vázquez B., N. A. Ruiz T., V. M. Zamora V. y F. Ramírez G. (2010) Producción de semilla de calabacita italiana (Cucurbita pepo L.) con acolchados plásticos fotoselectivos. Revista Fitotecnia Mexicana 33:265-270.

SAS Institute (2002) SAS/STAT Users' Guide. Versión 9.1. SAS Institute. Cary, North Carolina, USA. 5136 p.

SIAP, Servicio de Información Agroalimentaria y Pesquera (2018) Cierre de la producción agrícola por cultivo. Secretaria de Agricultura, Ganadería, Desarrollo Rural, Pesca y Alimentación. Ciudad de México. https://nube.siap.gob.mx/cierreagricola/ (Junio 2018)

SNICS, Servicio Nacional de Inspección y Certificación de Semillas (2018) Catálogo Nacional de Variedades Vegetales. 3er Trimestre 2018. Secretaria de Agricultura, Ganadería, Desarrollo Rural, Pesca y Alimentación. Ciudad de México. 42 p.

SNICS, Servicio Nacional de Inspección y Certificación de Semillas (2014) Tomate (Physalis spp.). Regla para la calificación de semilla de tomate. Servicio Nacional de Inspección y Certificación de Semillas, Secretaria de Agricultura, Ganadería, Desarrollo Rural, Pesca y Alimentación. México, D. F. 15 p. 